\title{
Bone Marrow Mesenchymal Stem Cells Transplantation Combined with Exercise Training Synergistically Promoted Functional Recovery After Spinal Cord Injury.
}

\section{Xin Sun}

Sichuan University West China Hospital https://orcid.org/0000-0003-3845-4342

\section{Liyi Huang}

Sichuan University West China Hospital

\section{Chenying Fu}

Sichuan University West China Hospital

\section{Lijuan Li}

Sichuan University West China Hospital

\section{Lu Wang}

Sichuan University West China Hospital

\section{Gaiqin Pei}

Sichuan University West China Hospital

\section{Yang Wang}

Sichuan University West China Hospital

\section{Qing Zhang}

Sichuan University West China Hospital

\section{Hongxin Cheng}

Sichuan University West China Hospital

\section{Chengqi He}

Sichuan University West China Hospital

Quan Wei ( $D$ weiquan@scu.edu.cn)

Sichuan University West China Hospital

\section{Research}

Keywords: spinal cord injury, bone marrow mesenchymal stem cells, exercise training, axon growth Posted Date: September 17th, 2021

DOl: https://doi.org/10.21203/rs.3.rs-880133/v1 
License: (c) (i) This work is licensed under a Creative Commons Attribution 4.0 International License. Read Full License 


\section{Abstract}

Background: Spinal cord injury (SCl) typically results in a devastating loss of neurological function below the level of injury. Although many strategies show considerable potential for $\mathrm{SCl}$ treatment, the therapeutic efficacy is limited. Here, we used a mouse model of thoracic contusive $\mathrm{SCl}$ to investigate whether the combination of bone marrow mesenchymal stem cells (BMMSCs) transplantation and exercise training has a synergistic effect on functional restoration.

Methods: BMMSCs were injected directly into the contusion epicenter immediately after $\mathrm{SCl}$, and the mice started treadmill training (TMT) 3 days after SCl. Locomotor function was evaluated by the Basso Mouse Scale (BMS), horizontal ladder test, and footprint analysis. Histological examination, transmission electron microscopy observation, immunofluorescence staining and western blotting were performed 8 weeks after $\mathrm{SCl}$ to further explore the potential mechanism of the synergistic repair effect.

Results: The combination of BMMSCs transplantation and TMT showed the best therapeutic effect on motor function recovery compared with the other treatment groups. Further investigations revealed that the combination of BMMSCs transplantation and TMT markedly reduced fibrotic scar tissue, protected neurons, promoted axon and myelin regeneration, and increased synapse formation to a larger extent than either TMT or BMMSCs transplantation alone. Additionally, the synergistic effects of BMMSCs transplantation and TMT on SCl recovery occurred via activation of the PI3K/AKT/mTOR pathway.

Conclusions: These findings suggest that BMMSCs transplantation combined with exercise training represents a promising combinatorial strategy to facilitate clinically meaningful recovery after $\mathrm{SCl}$.

\section{Introduction}

Spinal cord injury ( $\mathrm{SCl})$ is a serious and complex disease that can cause sensory and motor loss below the neurological level of injury [1,2]. According to quality country-level incidence studies of SCl, approximately 250,000 to 500,000 new cases occur each year $[3,4]$. Severe SCI typically leads to lifelong disability and enormous health care burdens [5]. The pathophysiology of spinal cord injury can be divided into primary and secondary injuries [6].The primary injury is the initial trauma to neurons, glial cells and their surrounding vasculature caused by initial mechanical forces such as compression, shearing, and stretching [7]. After the primary injury event, a cascade of secondary events, including apoptosis of neurons, glial scarring, demyelination, excitotoxicity, and the release of proinflammatory factors, can further exacerbate neurological dysfunction and expand the zone of tissue injury [8].

To date, many neuroregenerative strategies have been investigated as potential therapies for SCl. Previous studies have demonstrated that transplantation of bone marrow mesenchymal stem cells (BMMSCs) shows considerable potential for promoting tissue repair and functional improvement following SCI [9-12]. The most common mechanisms include neuroprotection, immunomodulation, neuronal relay formation, axonal regeneration, and remyelination $[13,14]$. Although BMMSCs show therapeutic promise according to preclinical findings, clinical trials still fail to demonstrate functional 
recovery and neural circuit restoration [15]. A randomized study tested the clinical efficacy of autologous BMMSCs transplantation in subacute SCl patients, and the results showed that BMMSCs transplantation into the injury site of SCl patients can be performed safely, but effects on motor function have not yet been observed [16]. Many other clinical trials have also failed to report satisfactory clinical improvements [17-19]. Stem cell grafts alone showed weak efficacy in functional recovery in human SCl. One possibility is that the transplanted cells could not form a sufficient number of functional connections and failed to sustain stable neuronal relays with the host circuitry for a long time [20]. Rehabilitation training seems to be an effective approach for locomotor function recovery after $\mathrm{SCl}$, which may be related to the plasticity of spinal neurons below the level of injury, corticospinal tract growth and enhancement of neurotrophic factor production [21-28]. Studies in clinical trials have also shown that functional recovery after exercise is related to the degree of activation of the motor cortex [29-31]. The permanent loss of function after $\mathrm{SCl}$ is caused by interruption of the ascending sensation and descending input of the spinal cord as well as failure of axon regeneration and neural circuit reconstruction [32]. A large body of evidence shows that the formation and remodeling of functional neural circuits depends on strengthening neuroplasticity $[33,34]$. In summary, BMMSC grafts or rehabilitation alone may target a single aspect of $\mathrm{SCl}$. Therefore, the combination of cell transplantation and exercise training may be more effective than individual therapy in SCl treatment.

The purpose of this study was to determine whether the combination of BMMSCs transplantation together with exercise training can improve the therapeutic efficacy of a single treatment in mice after thoracic contusive $\mathrm{SCl}$, and to investigate the potential mechanisms of combinatorial therapies in $\mathrm{SCl}$ repair.

\section{Materials And Methods}

\subsection{BMMSCs isolation and identification}

Four-week-old mice were sacrificed by cervical dislocation, and the femur and tibia were obtained with sterile forceps and surgical scissors. After the ends of the long bones were cut away, the bone marrow cavity was rinsed with minimal essential medium (alpha-MEM, Gibco, CA, USA) using a 1-ml syringe. The obtained cells were inoculated into tissue culture flasks and cultured in a humidified incubator at $37^{\circ} \mathrm{C}$ with $5 \% \mathrm{CO} 2$. Nonadherent cells were removed by changing the media, and the remaining adherent cells were passaged at $70-80 \%$ confluence. BMMSCs of passages $3-5$ were used in this study. Flow cytometry analysis (LSRFortessa, BD Bioscience) was used for BMMSCs identification. Passage 3 cells were stained with the following fluorescent-conjugated antibodies: PE-CD29, FITC-CD34, APC-CD44, and PerCP/CY5.5-CD45.

\subsection{Animals and SCI model}

Sixty-five 8-week-old female C57BL/6 mice were purchased from Chengdu Dossy Inc (China). The mice were housed under specific pathogen-free (SPF) conditions in the West China Experimental Animal Center at Sichuan University. The animal study was approved by the Laboratory Animal Ethics Committee of the 
West China Hospital of Sichuan University, and all animal procedures were conducted according to Chinese national guidelines for the care and use of laboratory animals (Ethical approval number: 20211198A).

The mice were first anesthetized with $3 \%$ isoflurane for induction, and then anesthesia was maintained under $1.5 \%$ isoflurane during all surgical procedures. After laminectomy at T10, the mice were subjected to contusive $\mathrm{SCl}$ at the same level using an Infinite Horizon impactor (70 kilodyne, Precision Systems \& Instrumentation). After suturing the surgical incisions, penicillin (50,000 U/kg/day) was administered intramuscularly for 3 days, and manual bladder expression was performed twice a day until spontaneous voiding returned. The sham group $(n=12)$ underwent only laminectomy, and the spinal cord remained intact. Forty-eight surviving $\mathrm{SCl}$ mice were randomized to the control, TMT, BMMSCs, and combination therapy (BMMSCs + TMT) groups ( $\mathrm{n}=12$ each).

\subsection{BMMSCs transplantation}

After SCl, $5 \mu$ l of phosphate-buffered saline (PBS) or BMMSCs $\left(1 \times 10^{5}\right.$ cells) was injected directly into the contusion epicenter at a depth of $1 \mathrm{~mm}$ using a Hamilton syringe positioned with a stereotaxic instrument (RWD Life Science, China) and driven by a syringe pump (KDS LEGATO 130, KD Scientific, USA) at a flow rate of $1 \mu \mathrm{l} / \mathrm{min}$.

\subsection{Treadmill training}

Three days of exercise was implemented in all groups prior to SCI injury for acclimatization. Treadmill training began 3 days after SCl. The mice in the TMT and BMMSCs + TMT group were allowed to run on the treadmill apparatus (ZH-PT, China) for 8 weeks ( $20 \mathrm{~min} /$ day, 6 days per week). The training speed was started at $4 \mathrm{~m} / \mathrm{min}$ and gradually increased according to the tolerance of the mice (the maximum speed was $9 \mathrm{~m} / \mathrm{min})$.

\subsection{Behavioral evaluation}

Locomotor recovery was evaluated by the Basso Mouse Scale (BMS), horizontal ladder test, and footprint analysis [35-37].

The BMS was assessed by two observers who were blinded to the experimental treatments before injury, 1 day after the injury, and then weekly after injury in an open field. The BMS was mainly used to determine the particular behavioral components of locomotion, such as trunk stability, plantar stepping, coordination, and paw position.

The horizontal ladder test requires adequate sensorimotor function to contact and feel the ladder rungs when stepping; thus, it is more sensitive to locomotor deficits [38]. The ladder consisted of 30 metal rungs (2 $\mathrm{mm}$ in diameter) spaced regularly $(1.3 \mathrm{~cm}$ apart) measuring $30 \mathrm{~cm}$ in height. Mice were exposed to the horizontal ladder at least once prior to surgery for acclimatization to the testing procedure. The walking ability of each mouse across the 30 metal rungs was recorded by video during each testing period. The 
hindlimb error rate (wrong steps/total steps) was evaluated weekly by a blinded investigator. A correct step corresponded to plantar or toe placements on rungs without slipping or dragging.

For the footprint analysis, mouse forepaws and hindlimbs were painted with red and black dyes, respectively. Mice were then allowed to walk in a straight path lined with white paper.

\subsection{Magnetic resonance imaging (MRI)}

T2-weighted sagittal magnetic resonance images were acquired using a 7T small animal MR system, Biospec USR70/30 (Bruker Corporation, Karlsruhe, Germany). During MRI, the mice were anesthetized by inhalation of $3 \%$ isoflurane and maintained under anesthesia by inhalation of $1.5 \%$ isoflurane through a face mask. The parameters were as follows: repetition time $(T R)=3000 \mathrm{~ms}$, echo time $(T E)=27 \mathrm{~ms}$, field of view $=40 \times 30 \mathrm{~mm}$, number of averages $=2$, matrix size $=256 \times 256$, slice number $=10$, slice thickness $=0.8 \mathrm{~mm}$, and slice gap $=0 \mathrm{~mm}$. Six animals were observed for each group. The lesion area was measured manually using Image $J$ software in each slice, and lesion volumes were calculated by summing lesion areas in each slice multiplied by the thickness of the slice.

\subsection{Histological examination}

Eight weeks after intervention, the animals were deeply anesthetized with isoflurane and transcardially perfused with saline followed by $4 \%$ paraformaldehyde in $0.1 \mathrm{M}$ phosphate buffer ( $\mathrm{pH}$ 7.2). Spinal cords ( $1 \mathrm{~cm}$ long for longitudinal sections and $0.5 \mathrm{~cm}$ long for transverse sections) centered at the injury epicenter were dissected and fixed in $4 \%$ neutral buffered paraformaldehyde for $24 \mathrm{~h}$, embedded in paraffin and cut into 2.5- $\mu$ m-thick slices. Longitudinal sections of the injured spinal cord were stained with Masson's trichrome to assess scar formation and with hematoxylin and eosin (H\&E) for morphological detection ( $\mathrm{n}=3$ per group). Transverse sections were stained with Nissl (cresyl violet) to count neurons after injury ( $\mathrm{n}=3$ per group).

\subsection{Transmission electron microscopy (TEM)}

Spinal cord tissue samples were collected, and transverse sections of the spinal cord were fixed in $2.5 \%$ glutaraldehyde in $0.1 \mathrm{M} \mathrm{PBS}$ at $4^{\circ} \mathrm{C}$ for $24 \mathrm{~h}$. Then, samples were postfixed in $1 \%$ osmium tetroxide in PBS, dehydrated in graded alcohols, and embedded in epoxy resin (Epon 812). Ultrathin sections of 50 $\mathrm{nm}$ were stained with uranyl acetate and lead citrate and finally examined with a JEM-1400 transmission electron microscope (JEOL, Tokyo, Japan).

\subsection{Western blot analysis}

The animals were sacrificed 8 weeks following injury, and spinal cord tissue at the injury epicenter was harvested for western blot analysis with $n=3$ mice per group. Homogenates from spinal cord tissue were lysed in radioimmunoprecipitation assay (RIPA) buffer (Beyotime Biotechnology, Shanghai, China) containing protease and phosphatase inhibitor cocktails at $4^{\circ} \mathrm{C}$. Protein concentrations were determined by using a bicinchoninic acid (BCA) kit (Beyotime). After normalization, samples were run on $10 \%$ SDSPAGE and transferred to a PVDF membrane. Membranes were blocked for 1 hour at room temperature 
and incubated with a specific primary antibody at $4^{\circ} \mathrm{C}$ overnight. After washing with Tris-buffered saline plus Tween (TBST), the membranes were incubated with anti-rabbit immunoglobulin G (IgG) horseradishperoxidase-conjugated secondary antibodies for $1 \mathrm{~h}$. Immunoreactive bands were visualized using an enhanced chemiluminescent detection system (Bio-Rad, USA). The immunoreactive bands were quantified by densitometric analysis using ImageJ Software. The primary antibodies used in this study included glial fibrillary acidic protein (GFAP), myelin basic protein (MBP), neuronal nuclei (NeuN), neurofilament-200 (NF200), postsynaptic density protein (PSD), synaptophysin (SYN), brain-derived neurotrophic factor (BDNF), vascular endothelial growth factor (VEGF), and p-mTOR, mTOR, and PTEN antibodies (all from Proteintech, China), AKT (Protein kinase B), p-AKT, PI3K (Phosphatidylinositol 3kinase), and p-PI3K antibodies (all from Cell Signaling Technology, USA), and nerve growth factor (NGF) antibody (from Abcam, USA).

\subsection{Immunofluorescence}

Spinal cord tissue was prepared as described previously and sectioned in the longitudinal or transverse plane at a thickness of $2.5 \mu \mathrm{m}$. Sections were deparaffinized and rehydrated with xylene $2 \times 10 \mathrm{~min}, 100 \%$ ethanol $2 \times 5 \mathrm{~min}$, and $96 \%$ ethanol, $80 \%$ ethanol, $70 \%$ ethanol, and $\mathrm{H} 2 \mathrm{O}$ for $5 \mathrm{~min}$ each. Antigen retrieval was performed with citrate buffer ( $\mathrm{pH} 6.2)$, and the sections were blocked with $10 \%$ goat serum. Sections were then incubated at $4^{\circ} \mathrm{C}$ overnight with the following primary antibodies: GFAP, MBP, NeuN, NF200, PSD, and SYN antibodies (all from Proteintech, China) and NGF antibody (from Abcam, USA). After washing, sections were then stained for $1 \mathrm{~h}$ at $37^{\circ} \mathrm{C}$ with the following fluorescent secondary antibodies: fluorescein isothiocyanate (FITC)-conjugated and rhodamine (TRITC)-conjugated secondary antibody (Proteintech, China). Nuclei were visualized by staining with DAPI. Images were acquired using an NSTORM \& A1 (Nikon, Tokyo, Japan) microscope.

\subsection{Statistical analysis}

Data were expressed as the means \pm SEM. Statistical analysis was performed using Prism 8.0 (GraphPad Software Inc., San Diego, CA). BMS scores and hindlimb error rates were analyzed using two-way analysis of variance (ANOVA) followed by Tukey's test for multiple comparisons. All other experiments were analyzed with one-way ANOVA followed by Tukey's post hoc multiple comparison test. A $P$-value $\leq$ 0.05 was considered significant.

\section{Results}

\subsection{Identification of BMMSCs}

After 3 passages, the cell surface markers of BMMSCs were determined by flow cytometry. The results showed positive expression (>99\%) of CD29 and CD44 and negative expression ( $<1 \%$ ) of CD34 and CD45, which indicated the high purity of the extracted BMMSCs (Additional file 1: Figure S1). 


\subsection{Combination therapy enhances locomotor function after $\mathrm{SCl}$}

BMS score assessment, and horizontal ladder and footprint analyses were then performed to evaluate the functional recovery of mice subjected to the different treatments. On the first day after injury, BMS assessment showed that the hindlimbs of all mice were completely paralyzed (BMS score $=0$ ), and then the mice showed different extents of recovery (Fig. 1A). Starting at 3 weeks after injury, the extent of functional recovery began to reveal the differences between the control group and the treatment groups (TMT, BMMSCs, BMMSCs + TMT; $p<0.001, p<0.001, p<0.001$, respectively). This difference remained statistically significant up to 8 weeks after $\mathrm{SCl}$. The significant difference between the BMMSCs group and the BMMSCs + TMT group emerged 6 weeks after SCl and persisted until the end of the trial $(\mathrm{p}<$ 0.05). In addition, a statistically significant difference appeared at 7 weeks and persisted at 8 weeks after injury between the TMT group and the BMMSCs + TMT group $(p<0.05, p<0.05$, respectively). The footprint test intuitively reflected differences in the tracks of hindlimbs among different groups. Mice in the control group showed obvious dragging of hindlimbs (blue ink) compared with the clear footprints of mice in the sham group, whereas mice in the BMMSCs + TMT group displayed fairly consistent hindlimb track with a few stumbled walking trajectories at 8 weeks after SCI (Fig. 1B). Animals in the TMT or BMMSCs group partially recovered coordination of the front and hind limbs but still showed partial drag. We also measured fine differences in motor function using a horizontal ladder test (Fig. 1C). Over an 8week testing period, all mice subjected to SCl showed a progressive decrease in the error rates (Fig. 1D). The mean error rate was significantly lower in the BMMSCs + TMT group than in the control group at 4 weeks $(p<0.05)$ after injury, which persisted until 8 weeks after injury $(p<0.01)$. However, no difference was found between the TMT or BMMSCs alone group and the control group at any time point examined. Taken together, these results show that the combined intervention can better promote locomotor function recovery than individual therapy after $\mathrm{SCl}$.

\subsection{Combination therapy promotes histological repair of the damaged spinal cord and reduces scar formation}

We investigated the effect of the combined therapy on the structural repair of the injured spinal cord tissue. The lesion volume of the injured spinal cord was measured at 8 weeks after injury using T2weighted MRI (Fig. 2A). Compared with that in the control group, a significant reduction in lesion volume was found in mice that received treatment (TMT, BMMSCs, BMMSCs + TMT; $p<0.01, p<0.001, p<0.001$, respectively) (Fig. 2C). Nevertheless, the differences between the combined treatment and TMT or BMMSCs alone were not statistically significant. The histological morphology changes in the injured spinal cord at 8 weeks after injury were evaluated using H\&E staining (Fig. 2B). Consistent with our MRI results, the relative lesion area in the injured spinal cords treated with TMT, BMMSCs, and BMMSCs + TMT was significantly reduced ( $p<0.001, p<0.001, p<0.001$, respectively), with less damaged tissue than that in the control group (Fig. 2D). Masson's trichrome staining was also used to estimate scar formation at the site of injury (Fig. 2B). TMT or BMMSCs alone did not significantly affect the fibrotic 
area, whereas a significantly lower average percentage of fibrosis was observed in the BMMSCs + TMT group than in the control group $(42.57 \pm 9.69 \%$ vs. $100.00 \pm 7.82 \%, p<0.001)$ (Fig. 2E). These results suggest that TMT or BMMSCs alone can promote the preservation of spinal cord tissue, but no significant synergism was noted between them. Furthermore, only the combination of BMMSCs and TMT can result in a significant reduction in scar formation.

\subsection{Combination therapy protects neurons and enhances the production of neurotrophic factors following $\mathrm{SCl}$}

To determine the neuroprotective effect of intervention on SCl mice, we examined neuronal density in the anterior horn of the injured spinal cord at 8 weeks following $\mathrm{SCl}$ by double immunofluorescent staining for GFAP (an astrocyte marker) and NeuN (a neuronal marker) (Fig. 3A). SCl induced a significant decrease in NeuN-positive neurons in the anterior horn of the injured spinal cord. The immunofluorescence results showed that the relative density of NeuN in the BMMSCs + TMT group significantly increased compared with those in the control, TMT alone, and BMMSCs alone groups $(p<$ $0.001, p<0.05, p<0.05$, Fig. $3 C$ ). However, TMT or BMMSCs alone did not significantly affect neuronal viability. We also investigated whether the combination treatment resulted in enhanced production of nerve growth factor (NGF), which is a crucial neurotrophin for neuronal growth and survival (Fig. 3B). TMT or BMMSCs alone did not provide any obvious beneficial effects on NGF production. Only when the two strategies were combined was the NGF level at the injured epicenter region significantly increased compared with that in the control group $(p<0.001$, Fig. 3D). For Nissl staining, as shown in Fig. 3E, a large number of normal neurons in the sham group displayed an integrative and granular-like morphology, while in the control group, their numbers were significantly decreased, and most of them exhibited shrunken cell bodies and Nissl granule dissolution. A quantitative comparison of Nissl bodies showed that only the BMMSCs + TMT group exhibited a significant increase compared with the control group. in the number of surviving neurons ( $p<0.05$, Fig. 3F). Additionally, western blot analysis of the NeuN and NGF proteins further confirmed the above results (Fig. 3G, 3H, 3I). We also detected the protein levels of brain-derived neurotrophic factor (BDNF) and vascular endothelial growth factor (VEGF), but the differences among all groups were not statistically significant (Figure S2A, S2C, S2D). These observations indicated that the combination therapy could exert a protective effect on neurons and promote the repair of damaged tissue after $\mathrm{SCl}$.

\subsection{Combination therapy promotes axonal generation and remyelination}

The capacity of axon regeneration at the lesion center of the spinal cord was evaluated by double immunofluorescent staining for GFAP and NF200 (an axonal marker) (Fig. 4A, 4B). GFAP-positive astrocytes accumulated along the lesion border (Fig. 4A). Notably, more NF200-positive, injured axons crossed the lesion border in the BMMSCs + TMT group compared with the axons observed in the TMT or BMMSCs alone group and the control group ( $<<0.05, p<0.05, p<0.001$, respectively) (Fig. 4E). In 
addition, the expression of NF200 in the TMT or BMMSCs group was significantly increased compared with that in the control group $(p<0.05)$. Double immunofluorescent staining for GFAP and MBP (a myelin marker) was performed to determine the effect of the intervention on remyelination (Fig. 4C). We also detected the microstructure of myelin using TEM (Fig. 4D). The immunofluorescence results suggested that TMT or BMMSCs alone increased the expression of MBP $(p<0.05)$, but the combination of the two strategies did not result in superior improvement compared with TMT or BMMSCs alone (Fig. 4F). Furthermore, TEM analysis revealed that only the BMMSCs + TMT group had significantly increased myelin thickness, as quantified by the G-ratio ( $p<0.001$, BMMSCs + TMT versus control, Fig. 4G). Moreover, western blot results further confirmed the immunofluorescence findings of NF200 and MBP (Fig. $5 \mathrm{H}, 5 \mathrm{I}$ ). Overall, these data indicate that the combination therapy can exert a more obvious effect on axon regeneration and remyelination than TMT or BMMSCs alone.

\subsection{Combination therapy increases synaptic plasticity after $\mathrm{SCl}$}

Immunofluorescence staining of PSD (a presynaptic marker protein) and SYN (a postsynaptic marker protein) in the injured spinal cord was performed to evaluate synaptic function (Fig. 5A, 5B). The expression levels of PSD and SYN at the injured epicenter region were substantially increased in the BMMSCs + TMT group compared with the control group $(p<0.001 ; p<0.01)$. TMT or BMMSCs alone tended to increase the expression levels of PSD and SYN, but no significant difference was observed between the TMT or BMMSC alone group and the control group (Fig. 5C, 5D). Western blot analysis for PSD and SYN (Fig. 5E, 5F, 5G) also confirmed the above results, indicating that TMT and BMMSCs acted synergistically to enhance synaptic plasticity better than the individual interventions.

\subsection{BMMSCs transplantation combined with TMT synergistically augments $\mathrm{SCl}$ recovery through activation of the PI3K/AKT/mTOR pathway}

Studies have confirmed that the PI3K/AKT/mTOR signaling pathway is involved in the growth of central nervous system (CNS) axons during development [39, 40]. During CNS maturity, phosphatase and tensin homolog on chromosome 10 (PTEN) suppresses the activity of this signaling pathway, accounting for the failure of axon regeneration in the injured spinal cord[41]. To further determine whether the combinatorial therapies promote $\mathrm{SCl}$ recovery by targeted activation of the PI3K/AKT/mTOR pathway, we performed western blot analysis to detect the protein levels of the key molecules in this pathway (Fig. 6A). The results indicate that only the combination therapy significantly enhanced the expression of $\mathrm{p}-\mathrm{PI} 3 \mathrm{~K}, \mathrm{p}$-AKT and $p$-mTOR compared with the control group ( $p<0.05 ; p<0.05 ; p<0.001$, Fig. 6B, 6C, 6D). Silencing of PTEN, a negative regulator of $\mathrm{mTOR}$, was shown to promote axon growth in animal models after $\mathrm{SCl}$. In our study, although the expression level of PTEN in the BMMSCs + TMT group tended to slightly decrease compared with that in the control group, the differences were not statistically significant $(p>0.05$, Figure 
S2A, S2B). The above data revealed that BMMSCs implantation combined with TMT promoted axonal regeneration and neuroplasticity via the $\mathrm{PI} 3 \mathrm{~K} / \mathrm{AKT} / \mathrm{mTOR}$ signaling pathway.

\section{Discussion}

Although many therapeutic interventions have shown promise in treating $\mathrm{SCl}$, focusing on a single aspect of repair cannot facilitate successful and functional regeneration in patients following SCl. Therefore, a combination of various interventions addressing the multiple aspects of SCl pathology is likely required. In this study, we opted for the combinatorial approach of neuroprotection and rehabilitation, capitalizing on cell transplantation and functional sensorimotor training to promote nerve regeneration and functional recovery. Treatment targets for $\mathrm{SCl}$ that can improve functional recovery include reduction of secondary damage, replacement of lost cells, removal of inhibitory molecules, axon regeneration through targeting neuronal mechanisms, resupply of trophic support, remyelination of demyelinated axons and rehabilitation for circuit remodeling [42-44]. Thus, in the present study, multiple integrated evidence derived in vivo was performed to assess $\mathrm{SCl}$ recovery, including locomotor performance, histopathological lesions, scar formation, axon growth and synapse remodeling and myelin regeneration.

Our results indicated that the combined treatment with BMMSCs and TMT showed the best therapeutic effect on functional recovery compared with other groups. The enhanced motor functional recovery by the combination therapy can probably be explained as follows. The combination of BMMSCs and TMT markedly reduced fibrotic scar tissue, protected neurons, promoted remyelination and axonal generation, and increased synapse formation, all to a larger extent than either TMT or BMMSCs alone. More strikingly, although the BMS score of each single therapy was significantly higher than that of the control group at the end of the trial, the mean error rate of hindlimbs between TMT or BMMSCs alone and the control group was not statistically significant. A possible explanation is that stepping across the rungs requires precise foot placement and grasping, which may provide a challenge to mice with poor locomotor performance $[45,46]$. Additionally, we noted that both single therapy and the combined therapy greatly reduced the tissue damage assessed by MRI and H\&E staining, and the combined therapy did not obviously enhance these independent effects of each single therapy. Thus, physical exercise or cell transplantation alone can be reasonably considered to also be able to promote tissue preservation.

Stem cell transplantation can promote $\mathrm{SCl}$ repair and functional improvement by differentiating into neurons or glial cells to replace damaged cells and secreting a variety of neurotrophic factors to protect the injured tissue and enhance axon regeneration [47, 48]. BMMSCs are generally accepted to have the advantages of high biosafety, wide biological effects and low immunogenicity [49]. In this study, we also observed that BMMSCs can promote axon and myelin regeneration, which is similar to findings in previous studies $[13,47]$. Scar formation is a pivotal determinant in limiting axonal regeneration after $\mathrm{SCl}$ [50]. Reactive astrogliosis is typically associated with the formation of compact scar borders around the inflammatory core [51]. Moreover, border-forming astrocytes increase the deposition of chondroitin sulfate proteoglycans (CSPGs), the major matrix contents of the glial scar, which may pose a physical and chemical barrier to axon outgrowth [52-55]. Our present data have shown that only the BMMSCs+ 
TMT treatment can lead to a substantial decrease in scar formation, which may provide a significant contribution to axon regeneration in the injured spinal cord. Indeed, we found that the combination of BMMSCs and TMT led to the highest expression of neurofilaments in the injured spinal cord.

$\mathrm{SCl}$ leads to the disruption of neural connectivity, thus resulting in severe permanent neurological disability. Restoration of function relies on promoting the formation of new connections and circuits [55, 56]. The remodeling of functional neural circuits in the spinal cord and brain may need to be driven by rehabilitation [57]. Combined treatments targeting the promotion of neuronal plasticity seem to be an effective approach. Current reports on the role of cell transplantation with exercise training are limited to several preclinical studies. A study in rats reported no evidence of functional recovery after bone marrow stromal cell transplantation or physical exercise alone or after both treatments [58]. In a subsequent chronic SCl mouse study, neural stem cell transplantation combined with TMT treatment was shown to significantly enhance functional recovery and facilitate neuronal differentiation of transplanted cells compared with either treatment alone [59]. Another recent study reported the functional and morphological benefits of a combinatorial approach with BMMSCs and early TMT treatment in a compression SCl mouse model [60]. Therefore, further investigations still need to explore the detailed mechanisms.

An intriguing finding in our study is that the BMMSCs + TMT group remarkably upregulated the $\mathrm{PI3K} /$ Akt/mTOR pathway. Mammalian target of rapamycin (mTOR), a serine/threonine protein kinase, can stimulate ribosomal protein translation [61]. Recent research has suggested that axonal growth in the injured CNS is mediated through PI3K/AKT/mTOR signalling pathway $[62,63]$. This pathway is suppressed in the injured CNS, which may limit the protein synthesis necessary for axon regeneration [64]. PTEN is considered to be an essential factor that can negatively regulate the PI3K/AKT/mTOR pathway, and genetic deletion of this molecule has been shown to increase the intrinsic growth capacity of neurons [65]. Phosphatidylinositol 3-kinases (PI3Ks) are a class of lipid kinases that convert phosphatidylinositol $(4,5)$-bisphosphate (PIP2) into phosphatidylinositol $(3,4,5)$-trisphosphate (PIP3) and then activate AKT and mTOR, ultimately mediating neuroprotection and axogenic protein synthesis [66]. After $\mathrm{SCl}$, the upregulation of PTEN can restrict the binding of AKTs to membranes by dephosphorylating PIP3 to PIP2, leading to the inactivation of the PI3K/AKT/mTOR pathway [40]. In our study, we also found that the expression of PTEN was upregulated in spinal cord tissues derived from mice subjected to SCl. We hypothesized that our interventions would reduce the expression of PTEN and then promote the activation of the PI3K/AKT/mTOR pathway. The results indicated that the combination group tended to downregulate the expression of PTEN, but the differences were not significant (Additional file 2: Figure S2A, S2B). One speculation is that the combinatorial approach of BMMSCs transplantation and exercise training may not only target PTEN to regulate the PI3K/AKT/mTOR signaling pathway. Notably, neurotrophic factors are crucial for supporting the viability of neurons and the growth of axons during mammalian CNS development [67]. Furthermore, the binding of neurotrophic factors to tyrosine kinase receptors (Trk) triggers their dimerization and autophosphorylation of tyrosine residues within the intracellular kinase domain, which can activate the PI3K/AKT/mTOR intracellular signaling pathway [40]. As expected, the NGF level at the lesion center was significantly increased in the BMMSCs + TMT group 
(Fig. 3B, 3D, 3G, 3I). Based on these findings, we speculated that BMMSCs combined with TMT exerts a neuroprotective effect by elevating NGF levels and then activating PI3K/AKT/mTOR signaling.

Additionally, BDNF and VEGF levels were not obviously altered by either single therapy or combination therapy in this study (Figure S2A, S2C, S2D). However, previous studies demonstrated that both BMMSCs and TMT have the potential to increase BDNF levels in the injured spinal cord $[59,60]$. This discrepancy might be due to different time points being examined. Further studies will be required to identify the complex interactions between exercise training and cell transplantation and determine the best temporal window.

\section{Conclusions}

In summary, the findings of the present study indicate that BMMSCs translation combined with exercise training synergistically improved motor function in paralyzed hindlimbs and established favorable conditions for functional restoration after $\mathrm{SCl}$ from the following aspects: promoting axonal regeneration and remyelination, enhancing synaptic plasticity and neurotrophin secretion, suppressing scar formation, and protecting neurons. Taken together, this study presents a promising combinatorial therapy for patients suffering from SCl. In addition, further studies are required to identify and understand the complex interactions between therapies.

\section{Abbreviations}

SCl: spinal cord injury, BMMSCs: bone marrow mesenchymal stem cells, TMT: treadmill training, BMS: Basso Mouse Scale, MRI: Magnetic resonance imaging, H\&E: hematoxylin and eosin, TEM: Transmission electron microscopy, GFAP: glial fibrillary acidic protein, MBP: myelin basic protein, NeuN: neuronal nuclei, NF200: neurofilament-200, PSD: postsynaptic density protein, SYN: synaptophysin, BDNF: brain-derived neurotrophic factor, VEGF: vascular endothelial growth factor, NGF: nerve growth factor, PTEN: phosphatase and tensin homolog on chromosome 10, mTOR: Mammalian target of rapamycin, AKT: Protein kinase B, PI3K: Phosphatidylinositol 3-kinase.

\section{Declarations}

\section{Acknowledgments}

None.

\section{Funding}

This work was supported by the National Natural Science Foundation of China [Grant No. 81572231], and the Project of the Science and Technology Department in Sichuan province [Grant No. 2019YJ0119].

\section{Authors' Contributions}


X.S. and Q.W. conceived the idea and designed the research studies. X.S. and L.H. conducted the experiments. C.F., L.L., L.W., and G.P. participated in SCl model preparation and data analysis. Y.W., Q.Z., and H.C. acquired and analyzed the data. X.S. drafted the initial manuscript. Q.W. and C.H. reviewed and revised the manuscript. All authors approved the final version of manuscript.

\section{Availability of data and materials}

All data generated during this study are included in this article.

\section{Ethics approval and consent to participate}

All procedures were approved by the Laboratory Animal Ethics Committee of the West China Hospital of Sichuan University (Ethical approval number: 20211198A).

\section{Consent for publication}

Not applicable.

\section{Competing interests}

All authors declare no competing interests.

\section{References}

1. Perrouin-Verbe B, Lefevre C, Kieny P, Gross R, Reiss B, Le Fort M. Spinal cord injury: A multisystem physiological impairment/dysfunction. Rev Neurol (Paris). 2021,177(5): 594-605.

2. McDonald JW, Sadowsky C. Spinal-cord injury. Lancet. 2002,359(9304): 417-25.

3. International Perspectives on Spinal Cord Injury. World Health Organization.

4. Liu T, Xie S, Wang Y, Tang J, He X, Yan T, et al. Effects of App-Based Transitional Care on the SelfEfficacy and Quality of Life of Patients With Spinal Cord Injury in China: Randomized Controlled Trial. JMIR Mhealth Uhealth. 2021,9(4): e22960.

5. Karsy M, Hawryluk G. Modern Medical Management of Spinal Cord Injury. Curr Neurol Neurosci Rep. 2019,19(9): 65.

6. Tator $\mathrm{CH}$. Update on the pathophysiology and pathology of acute spinal cord injury. Brain Pathol. 1995,5(4): 407-13.

7. Ahuja CS, Nori S, Tetreault L, Wilson J, Kwon B, Harrop J, et al. Traumatic Spinal Cord Injury-Repair and Regeneration. Neurosurgery. 2017,80(3s): S9-s22.

8. Ahuja CS, Wilson JR, Nori S, Kotter MRN, Druschel C, Curt A, et al. Traumatic spinal cord injury. Nat Rev Dis Primers. 2017,3: 17018.

9. Lin L, Lin H, Bai S, Zheng L, Zhang X. Bone marrow mesenchymal stem cells (BMSCs) improved functional recovery of spinal cord injury partly by promoting axonal regeneration. Neurochem Int. 
2018,115: 80-84.

10. Chiba Y, Kuroda S, Maruichi K, Osanai T, Hokari M, Yano S, et al. Transplanted bone marrow stromal cells promote axonal regeneration and improve motor function in a rat spinal cord injury model. Neurosurgery. 2009,64(5): 991-9, discussion 99-1000.

11. Ding P, Yang Z, Wang W, Wang J, Xue L. Transplantation of bone marrow stromal cells enhances infiltration and survival of CNP and Schwann cells to promote axonal sprouting following complete transection of spinal cord in adult rats. Am J Transl Res. 2014,6(3): 224-35.

12. Ide C, Nakano N, Kanekiyo K. Cell transplantation for the treatment of spinal cord injury - bone marrow stromal cells and choroid plexus epithelial cells. Neural Regen Res. 2016,11(9): 1385-88.

13. Assinck P, Duncan GJ, Hilton BJ, Plemel JR, Tetzlaff W. Cell transplantation therapy for spinal cord injury. Nat Neurosci. 2017,20(5): 637-47.

14. Shende P, Subedi M. Pathophysiology, mechanisms and applications of mesenchymal stem cells for the treatment of spinal cord injury. Biomed Pharmacother. 2017,91: 693-706.

15. Cofano F, Boido M, Monticelli M, Zenga F, Ducati A, Vercelli A, et al. Mesenchymal Stem Cells for Spinal Cord Injury: Current Options, Limitations, and Future of Cell Therapy. Int J Mol Sci. 2019,20(11).

16. Karamouzian S, Nematollahi-Mahani SN, Nakhaee N, Eskandary H. Clinical safety and primary efficacy of bone marrow mesenchymal cell transplantation in subacute spinal cord injured patients. Clin Neurol Neurosurg. 2012,114(7): 935-9.

17. Mendonça MV, Larocca TF, de Freitas Souza BS, Villarreal CF, Silva LF, Matos AC, et al. Safety and neurological assessments after autologous transplantation of bone marrow mesenchymal stem cells in subjects with chronic spinal cord injury. Stem Cell Res Ther. 2014,5(6): 126.

18. Syková E, Homola A, Mazanec R, Lachmann H, Konrádová SL, Kobylka P, et al. Autologous bone marrow transplantation in patients with subacute and chronic spinal cord injury. Cell Transplant. 2006,15(8-9): 675-87.

19. Geffner LF, Santacruz P, Izurieta M, Flor L, Maldonado B, Auad AH, et al. Administration of autologous bone marrow stem cells into spinal cord injury patients via multiple routes is safe and improves their quality of life: comprehensive case studies. Cell Transplant. 2008,17(12): 1277-93.

20. Hutson TH, Di Giovanni S. The translational landscape in spinal cord injury: focus on neuroplasticity and regeneration. Nat Rev Neurol. 2019,15(12): 732-45.

21. Houle JD, Côté MP. Strategies and prospects of effective neural circuits reconstruction after spinal cord injury. Ann N Y Acad Sci. 2013,1279(1): 154-63.

22. Morawietz C, Moffat F. Effects of locomotor training after incomplete spinal cord injury: a systematic review. Arch Phys Med Rehabil. 2013,94(11): 2297-308.

23. Hinahon E, Estrada C, Tong L, Won DS, de Leon RD. Robot-Applied Resistance Augments the Effects of Body Weight-Supported Treadmill Training on Stepping and Synaptic Plasticity in a Rodent Model of Spinal Cord Injury. Neurorehabil Neural Repair. 2017,31(8): 746-57. 
24. Houle JD, Côté MP. Axon regeneration and exercise-dependent plasticity after spinal cord injury. Ann N Y Acad Sci. 2013,1279(1): 154-63.

25. Wang H, Liu NK, Zhang YP, Deng L, Lu QB, Shields CB, et al. Treadmill training induced lumbar motoneuron dendritic plasticity and behavior recovery in adult rats after a thoracic contusive spinal cord injury. Exp Neurol. 2015,271: 368-78.

26. Foret A, Quertainmont R, Botman O, Bouhy D, Amabili P, Brook G, et al. Stem cells in the adult rat spinal cord: plasticity after injury and treadmill training exercise. J Neurochem. 2010,112(3): 762-72.

27. Flynn JR, Dunn LR, Galea MP, Callister R, Callister RJ, Rank MM. Exercise training after spinal cord injury selectively alters synaptic properties in neurons in adult mouse spinal cord. J Neurotrauma. 2013,30(10): 891-6.

28. Sun T, Ye C, Wu J, Zhang Z, Cai Y, Yue F. Treadmill step training promotes spinal cord neural plasticity after incomplete spinal cord injury. Neural Regen Res. 2013,8(27): 2540-7.

29. Jurkiewicz MT, Mikulis DJ, Mcllroy WE, Fehlings MG, Verrier MC. Sensorimotor cortical plasticity during recovery following spinal cord injury: a longitudinal fMRI study. Neurorehabil Neural Repair. 2007,21(6): 527-38.

30. Fu J, Wang H, Deng L, Li J. Exercise Training Promotes Functional Recovery after Spinal Cord Injury. Neural Plast. 2016,2016: 4039580.

31. Jo HJ, Perez MA. Corticospinal-motor neuronal plasticity promotes exercise-mediated recovery in humans with spinal cord injury. Brain. 2020,143(5): 1368-82.

32. Orr MB, Gensel JC. Spinal Cord Injury Scarring and Inflammation: Therapies Targeting Glial and Inflammatory Responses. Neurotherapeutics. 2018,15(3): 541-53.

33. Torres-Espín A, Forero J, Fenrich KK, Lucas-Osma AM, Krajacic A, Schmidt E, et al. Eliciting inflammation enables successful rehabilitative training in chronic spinal cord injury. Brain. 2018,141(7): 1946-62.

34. Yang B, Zhang F, Cheng F, Ying L, Wang C, Shi K, et al. Strategies and prospects of effective neural circuits reconstruction after spinal cord injury. Cell Death Dis. 2020,11(6): 439.

35. Basso DM, Fisher LC, Anderson AJ, Jakeman LB, McTigue DM, Popovich PG. Basso Mouse Scale for locomotion detects differences in recovery after spinal cord injury in five common mouse strains. $J$ Neurotrauma. 2006,23(5): 635-59.

36. Cummings BJ, Engesser-Cesar C, Cadena G, Anderson AJ. Adaptation of a ladder beam walking task to assess locomotor recovery in mice following spinal cord injury. Behav Brain Res. 2007,177(2): 232-41.

37. Ma M, Basso DM, Walters P, Stokes BT, Jakeman LB. Behavioral and histological outcomes following graded spinal cord contusion injury in the C57BI/6 mouse. Exp Neurol. 2001,169(2): 239-54.

38. Soblosky JS, Song JH, Dinh DH. Graded unilateral cervical spinal cord injury in the rat: evaluation of forelimb recovery and histological effects. Behav Brain Res. 2001,119(1): 1-13. 
39. Li R, Li DH, Zhang HY, Wang J, Li XK, Xiao J. Growth factors-based therapeutic strategies and their underlying signaling mechanisms for peripheral nerve regeneration. Acta Pharmacol Sin. 2020,41(10): 1289-300.

40. Berry M, Ahmed Z, Morgan-Warren P, Fulton D, Logan A. Prospects for mTOR-mediated functional repair after central nervous system trauma. Neurobiol Dis. 2016,85: 99-110.

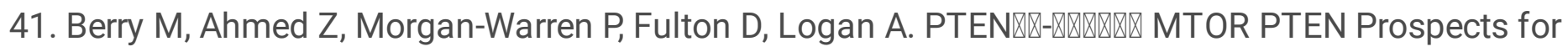
mTOR-mediated functional repair after central nervous system trauma. Neurobiol Dis. 2016,85: 99110.

42. Griffin JM, Bradke F. Therapeutic repair for spinal cord injury: combinatory approaches to address a multifaceted problem. EMBO Mol Med. 2020,12(3): e11505.

43. Führmann T, Anandakumaran PN, Shoichet MS. Combinatorial Therapies After Spinal Cord Injury: How Can Biomaterials Help? Adv Healthc Mater. 2017,6(10).

44. O'Shea TM, Burda JE, Sofroniew MV. Cell biology of spinal cord injury and repair. J Clin Invest.127(9): 3259-70.

45. Metz GA, Whishaw IQ. The ladder rung walking task: a scoring system and its practical application. J Vis Exp. 2009(28).

46. Emerick AJ, Kartje GL. Behavioral recovery and anatomical plasticity in adult rats after cortical lesion and treatment with monoclonal antibody IN-1. Behav Brain Res. 2004,152(2): 315-25.

47. Veneruso V, Rossi F, Villella A, Bena A, Forloni G, Veglianese P. Stem cell paracrine effect and delivery strategies for spinal cord injury regeneration. J Control Release. 2019,300: 141-53.

48. Vismara I, Papa S, Rossi F, Forloni G, Veglianese P. Current Options for Cell Therapy in Spinal Cord Injury. Trends Mol Med. 2017,23(9): 831-49.

49. Shao A, Tu S, Lu J, Zhang J. Crosstalk between stem cell and spinal cord injury: pathophysiology and treatment strategies. Stem Cell Res Ther. 2019,10(1): 238.

50. Gaudet AD, Popovich PG. Extracellular matrix regulation of inflammation in the healthy and injured spinal cord. Exp Neurol. 2014,258: 24-34.

51. Fitch MT, Doller C, Combs CK, Landreth GE, Silver J. Cellular and molecular mechanisms of glial scarring and progressive cavitation: in vivo and in vitro analysis of inflammation-induced secondary injury after CNS trauma. J Neurosci. 1999,19(19): 8182-98.

52. Canning DR, Höke A, Malemud CJ, Silver J. A potent inhibitor of neurite outgrowth that predominates in the extracellular matrix of reactive astrocytes. Int J Dev Neurosci. 1996,14(3): 153-75.

53. Hara M, Kobayakawa K, Ohkawa Y, Kumamaru H, Yokota K, Saito T, et al. Interaction of reactive astrocytes with type I collagen induces astrocytic scar formation through the integrin-N-cadherin pathway after spinal cord injury. Nat Med. 2017,23(7): 818-28.

54. Schachtrup C, Ryu JK, Helmrick MJ, Vagena E, Galanakis DK, Degen JL, et al. Fibrinogen triggers astrocyte scar formation by promoting the availability of active TGF-beta after vascular damage. $J$ Neurosci. 2010,30(17): 5843-54. 
55. Tran AP, Warren PM, Silver J. The Biology of Regeneration Failure and Success After Spinal Cord Injury. Physiol Rev. 2018,98(2): 881-917.

56. Onifer SM, Smith GM, Fouad K. Plasticity after spinal cord injury: relevance to recovery and approaches to facilitate it. Neurotherapeutics. 2011,8(2): 283-93.

57. Loy K, Bareyre FM. Rehabilitation following spinal cord injury: how animal models can help our understanding of exercise-induced neuroplasticity. Neural Regen Res. 2019,14(3): 405-12.

58. Yoshihara H, Shumsky JS, Neuhuber B, Otsuka T, Fischer I, Murray M. Combining motor training with transplantation of rat bone marrow stromal cells does not improve repair or recovery in rats with thoracic contusion injuries. Brain Res. 2006,1119(1): 65-75.

59. Tashiro S, Nishimura S, Iwai H, Sugai K, Zhang L, Shinozaki M, et al. Functional Recovery from Neural Stem/Progenitor Cell Transplantation Combined with Treadmill Training in Mice with Chronic Spinal Cord Injury. Sci Rep. 2016,6: 30898.

60. Massoto TB, Santos ACR, Ramalho BS, Almeida FM, Martinez AMB, Marques SA. Mesenchymal stem cells and treadmill training enhance function and promote tissue preservation after spinal cord injury. Brain Res. 2020,1726: 146494.

61. Song MS, Salmena L, Pandolfi PP. The functions and regulation of the PTEN tumour suppressor. Nat Rev Mol Cell Biol. 2012,13(5): 283-96.

62. Park KK, Liu K, Hu Y, Smith PD, Wang C, Cai B, et al. Promoting axon regeneration in the adult CNS by modulation of the PTEN/mTOR pathway. Science. 2008,322(5903): 963-6.

63. He Z, Jin Y. Intrinsic Control of Axon Regeneration. Neuron. 2016,90(3): 437-51.

64. Yin H, Shen L, Xu C, Liu J. Lentivirus-Mediated Overexpression of miR-29a Promotes Axonal Regeneration and Functional Recovery in Experimental Spinal Cord Injury via PI3K/Akt/mTOR Pathway. Neurochem Res. 2018,43(11): 2038-46.

65. Danilov CA, Steward O. Conditional genetic deletion of PTEN after a spinal cord injury enhances regenerative growth of CST axons and motor function recovery in mice. Exp Neurol. 2015,266: 14760.

66. Liu NK, Xu XM. Neuroprotection and its molecular mechanism following spinal cord injury. Neural Regen Res. 2012,7(26): 2051-62.

67. Castilla-Cortázar I, Iturrieta I, García-Magariño M, Puche JE, Martín-Estal I, Aguirre GA, et al. Neurotrophic Factors and Their Receptors Are Altered by the Mere Partial IGF-1 Deficiency. Neuroscience. 2019,404: 445-58.

\section{Figures}


A

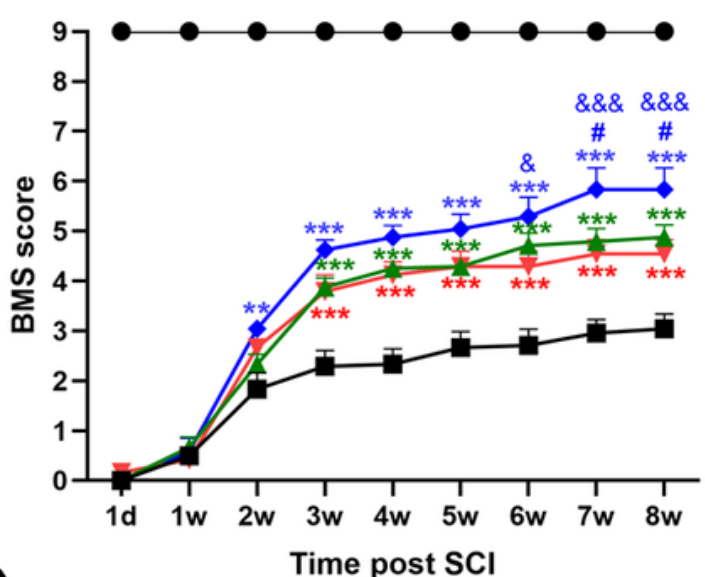

B

C
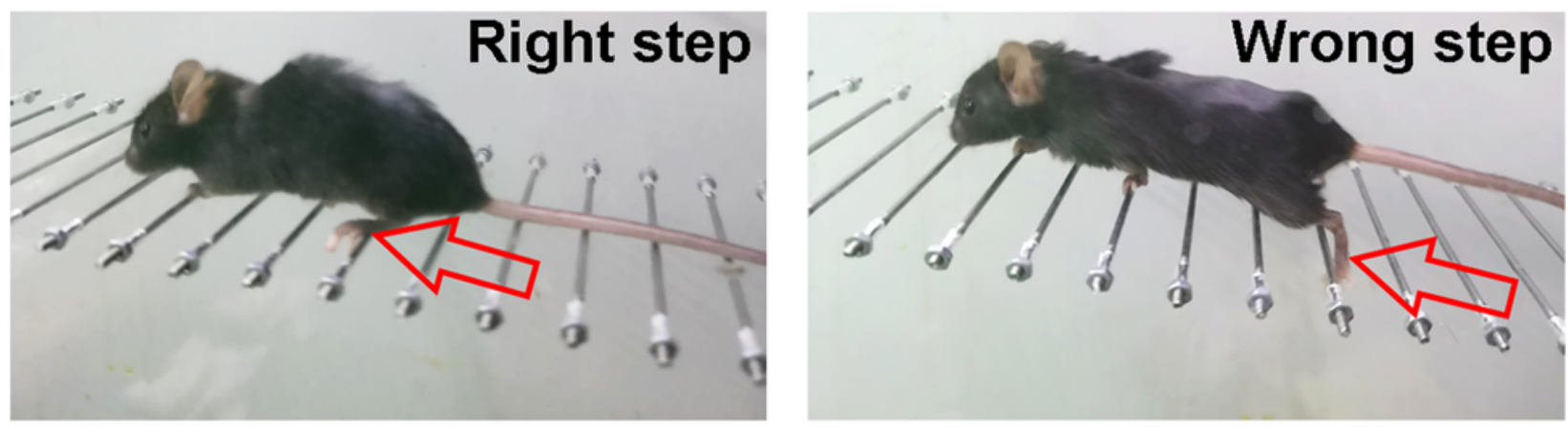

D

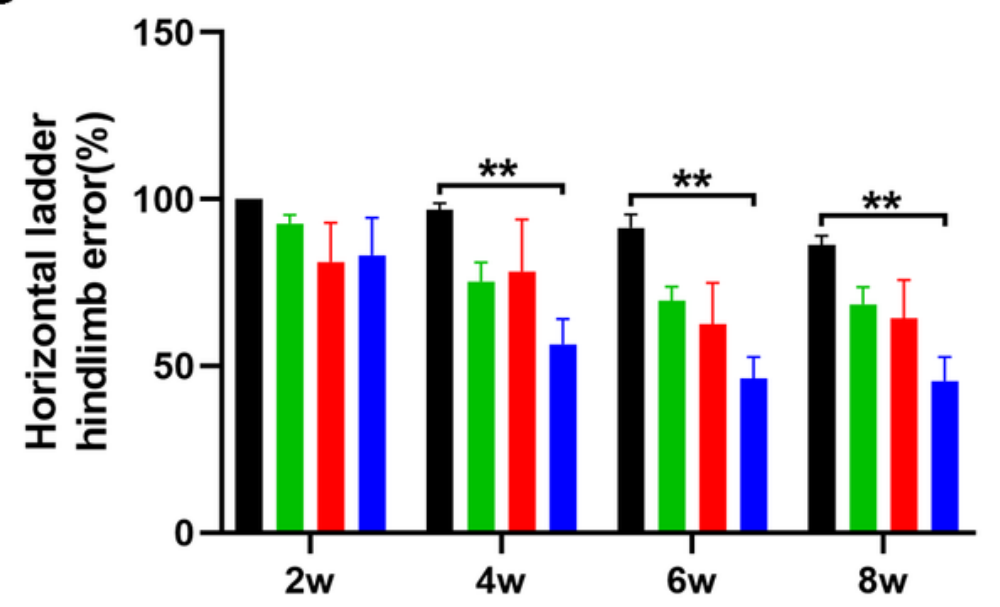

- Control

- TMT

BMMSCs

- BMMSCs+TMT

Figure1

\section{Figure 1}

Combining BMMSCs and TMT improved motor function after SCI. A. Basso Mouse Scale (BMS) locomotion scores over the 8-week period among different treatment groups. ${ }^{*} p<0.05,{ }^{\star \star} p<0.01$,

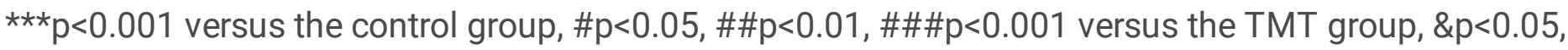
$\& \& p<0.01, \& \& \& p<0.001$ versus the BMMSCs group. $N=12$ per group. $B$. Footprint analysis of the different treatment groups 8 weeks post SCl. Red: front paw print, Blue: hind paw print. C. Representative 
photographs illustrating horizontal ladder. D. The horizontal ladder test at weeks $2,4,6,8$. ${ }^{*} p<0.05$, ${ }^{\star *} p<0.01$. Data in all figures represent mean \pm SEM (Two-way ANOVA), $N=5$ per group.

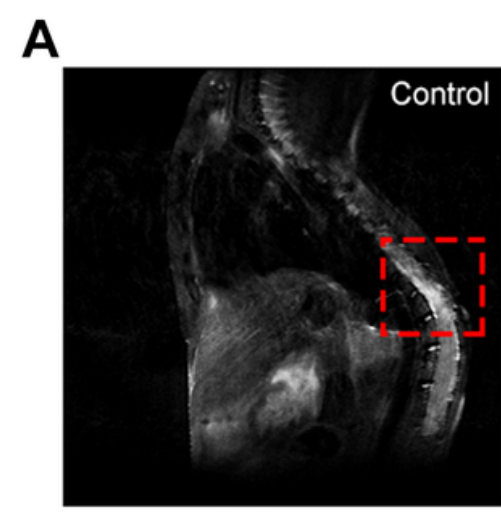

B
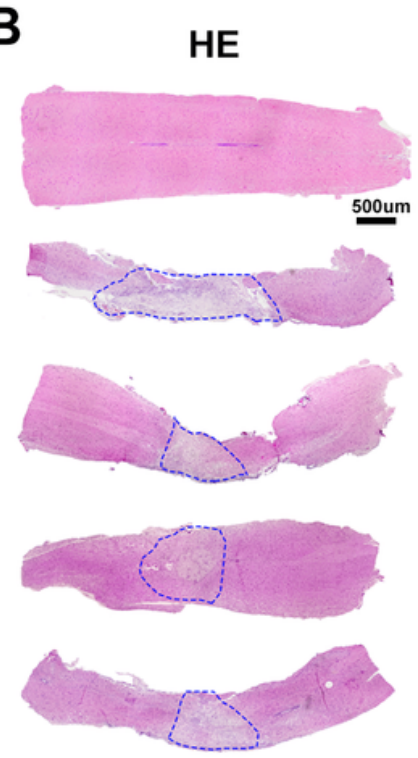

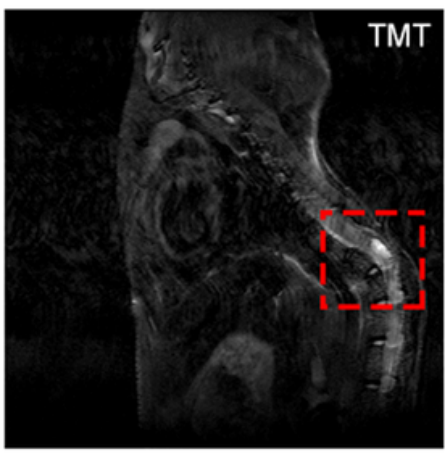

Masson

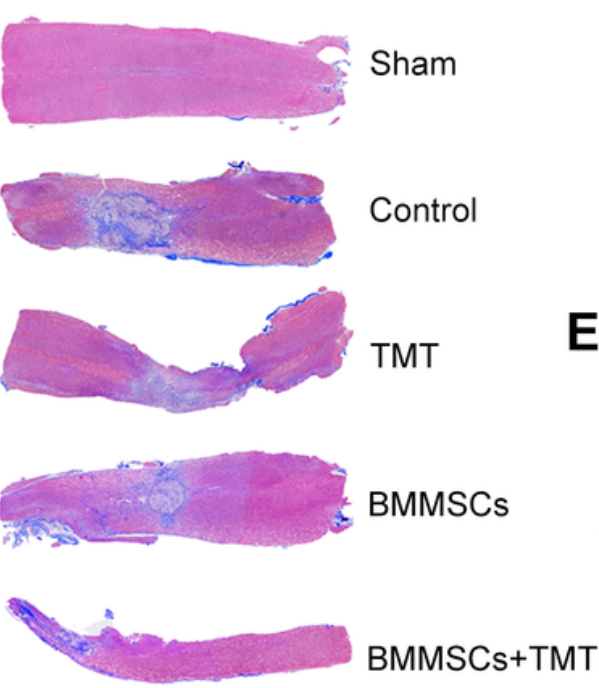

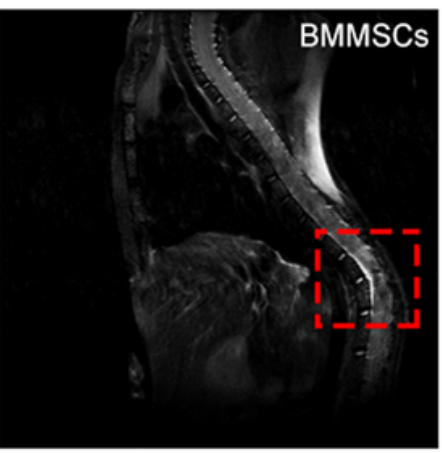

C
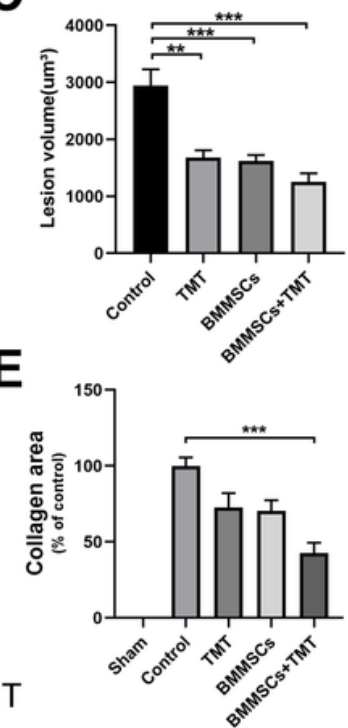

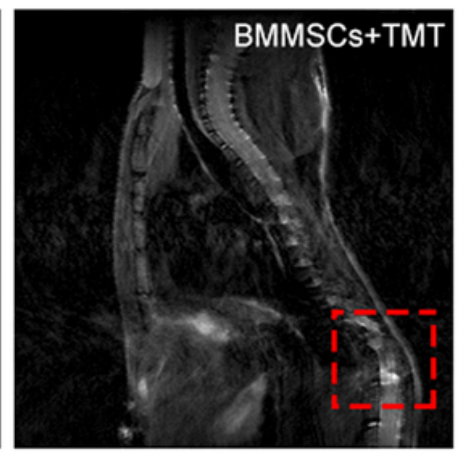

D

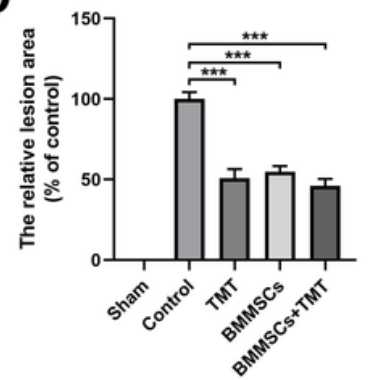

Figure2

\section{Figure 2}

Combining BMMSCs and TMT promoted the histological repair of the damaged spinal cord in SCl. A-B. $\mathrm{MRI}$ analysis, $\mathrm{HE}$ staining and Masson staining of the spinal cord at weeks 8 after $\mathrm{SCl}$ in different groups. C-E. Quantification of MRI, HE staining and Masson staining results in different groups. ${ }^{\star}{ }^{*} p<0.001 . N \geq 3$ per group for histology analysis. 
A
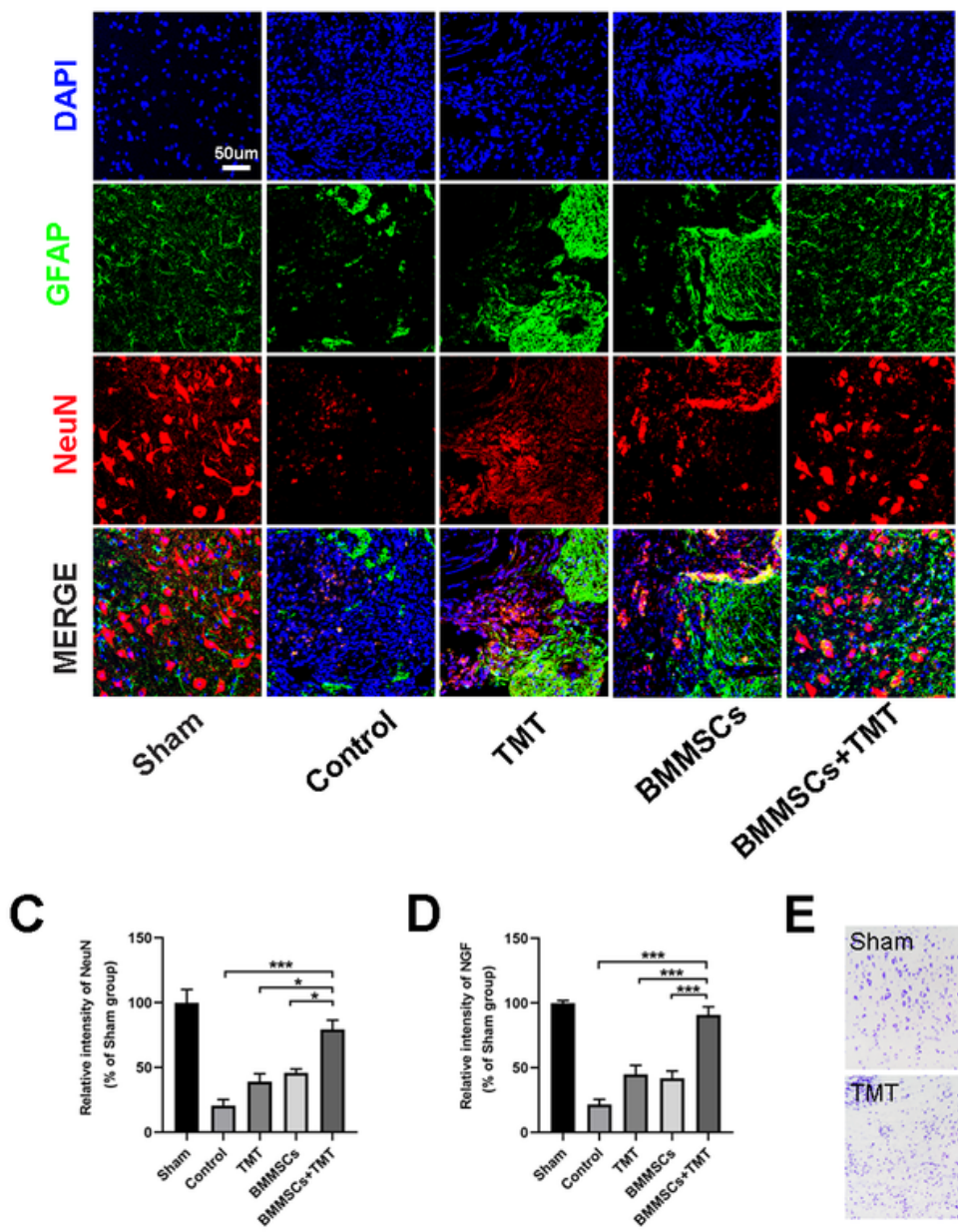

G

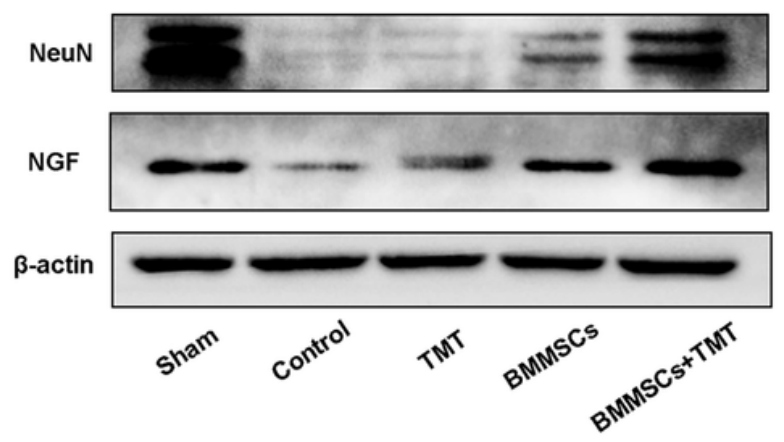

D

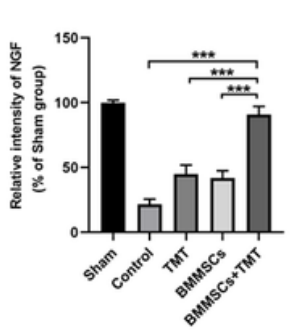

E
B
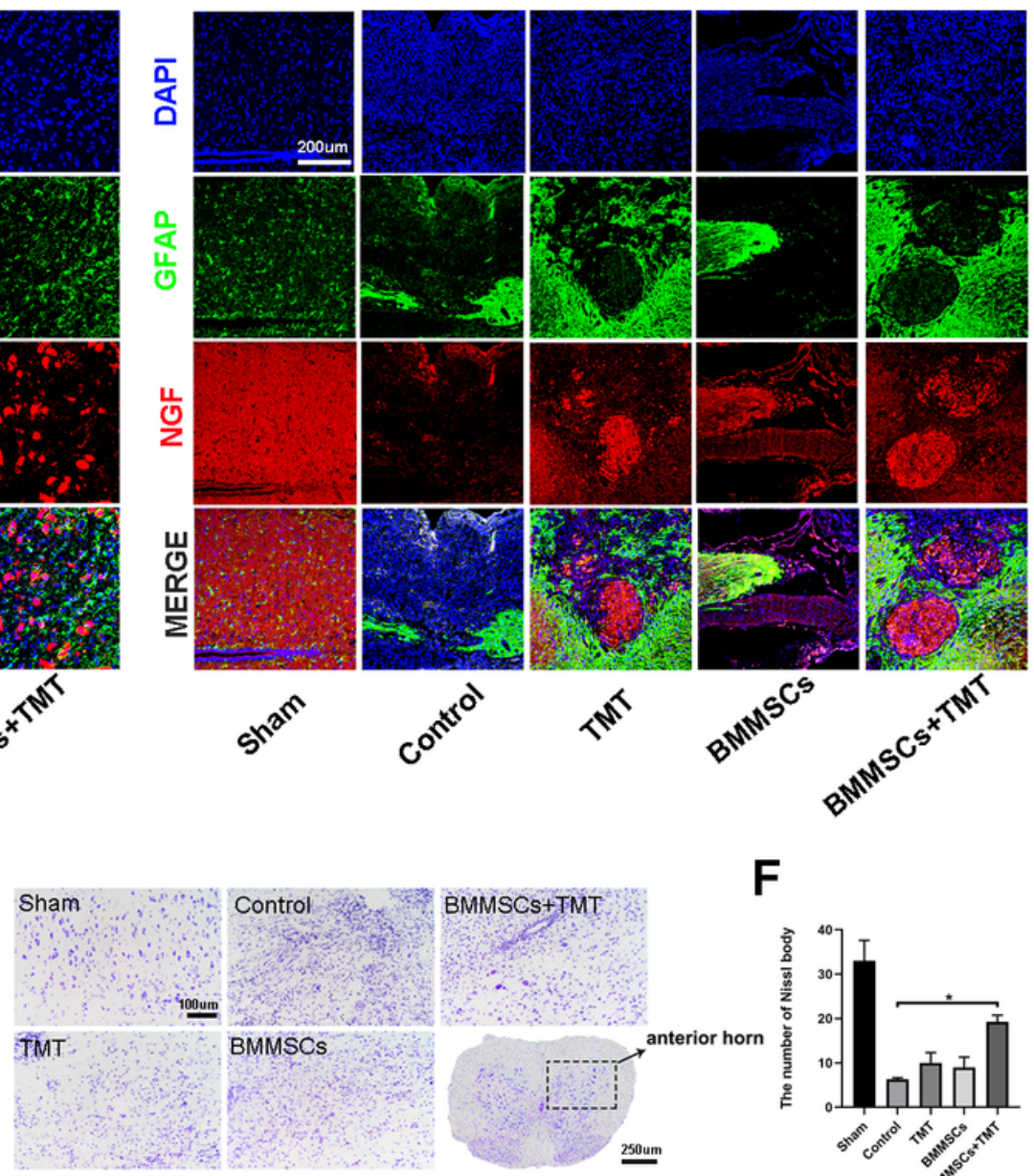

H

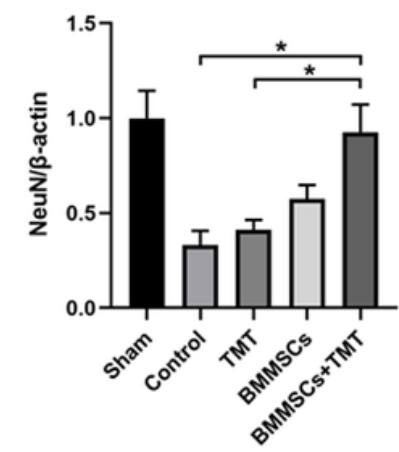

Figure3

\section{Figure 3}

Combining BMMSCs and TMT provided neuronal protection and enhanced the expression of neurotrophic factors. A. Representative images of NeuN staining from the anterior horn of injured spinal cord in the different groups at 8 weeks after SCl. GFAP_(green), NeuN_(Red), DAPI_(blue). B. Representative Images of immunofluorescence staining of NGF in the lesion epicenter. GFAP_(green), NGF_(Red), DAPI_(blue). C-D. Quantification data of NeuN and NGF positive expression in different groups. E. Representative images of Nissl staining from the anterior horn of spinal cord. F. Quantification 
of Nissl staining in different groups. G-I. Representative western blot images and quantification of NeuN and NGF in each group. ${ }^{*} p<0.05,{ }^{* *} p<0.01,{ }^{* *} p<0.001 . N=3$ per group.

A

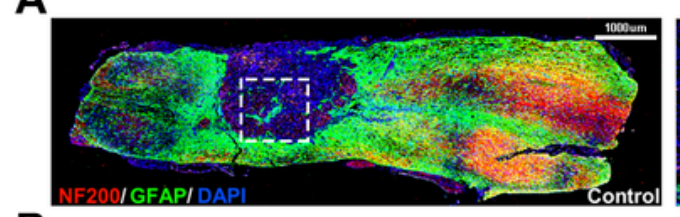

B

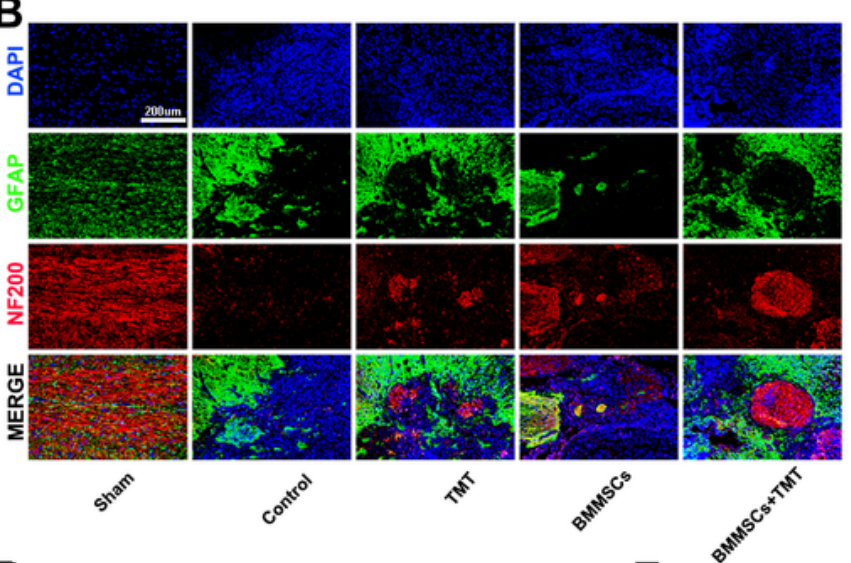

D

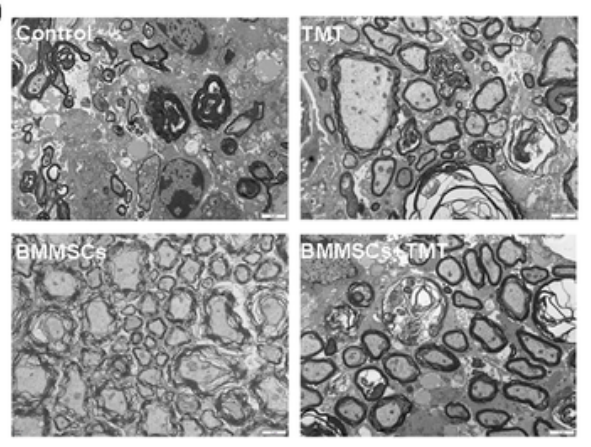

$\mathbf{H}$
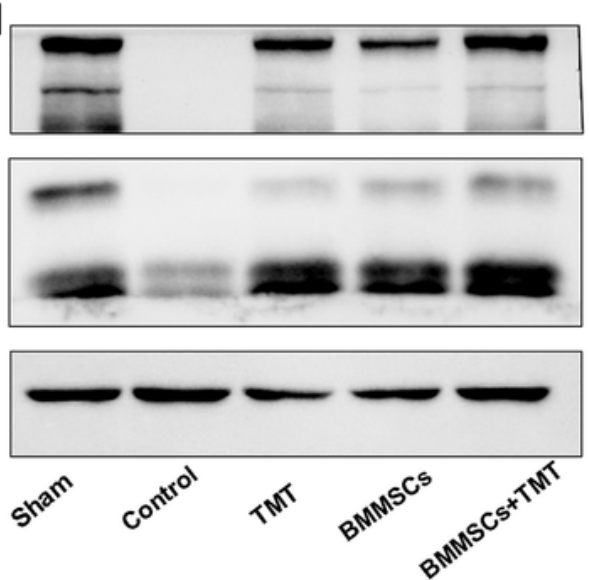

NF200
C

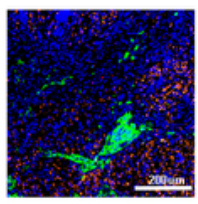

E

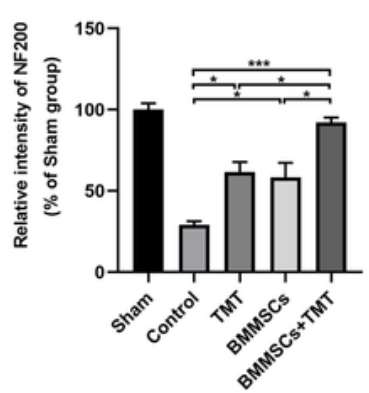

I
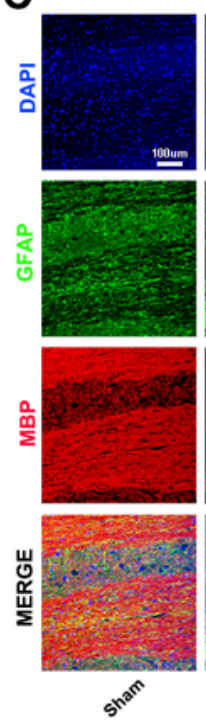

F
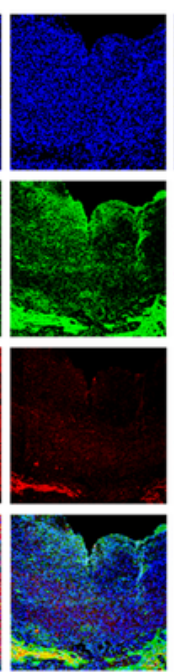

$0^{00}$
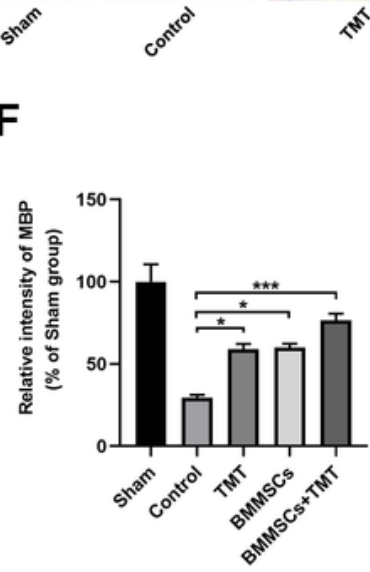

J

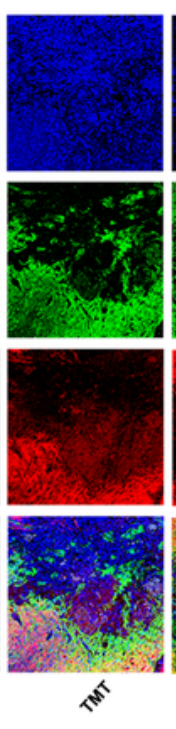

G
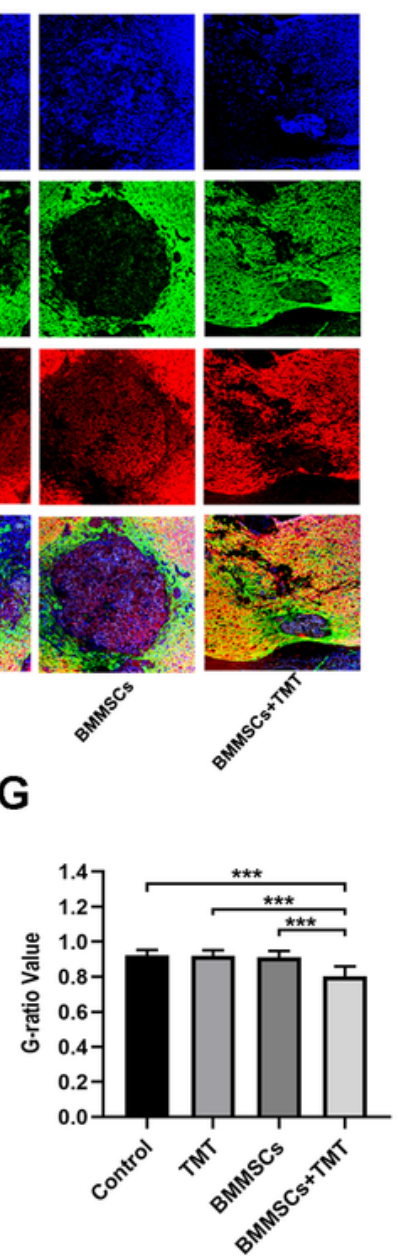

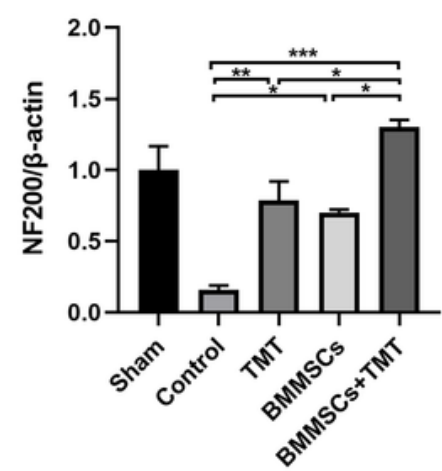

Figure4

\section{Figure 4}

Combining BMMSCs and TMT enhanced axon and myelin regrowth. A. Horizontal section immunostained with NF200_(red) and GFAP_(green) showing the distribution of NF200-positive axons in the control group. The square area is the injury site and is magnified in the right panel. B-C. 
Representative Images of immunofluorescence staining of NF200 (B) and MBP (C) in the lesion center 8 weeks after SCl. GFAP_(green), NF200_(red), MBP_(red), DAPI_(blue). D. Transmission electron microscope_(TEM) images of the myelin sheath in different groups 8 weeks after SCI. E-F. Quantification of immunofluorescence staining of NF200 and MBP. G. Quantification of myelin sheath thickness by gratio analysis. H-J. Representative western blot images and quantification of NF200 and MBP. ${ }^{\star} p<0.05$, $\star \star x p<0.01, * \star * p<0.001 . N=3$ per group.

A

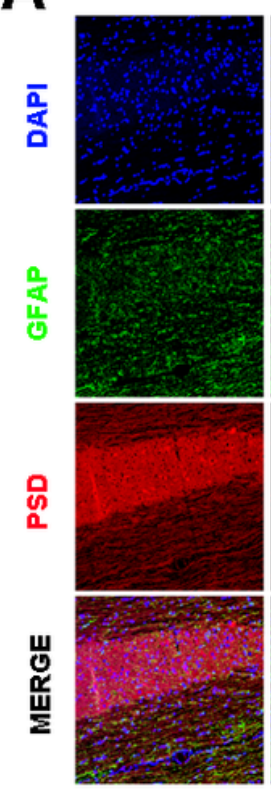

50

E
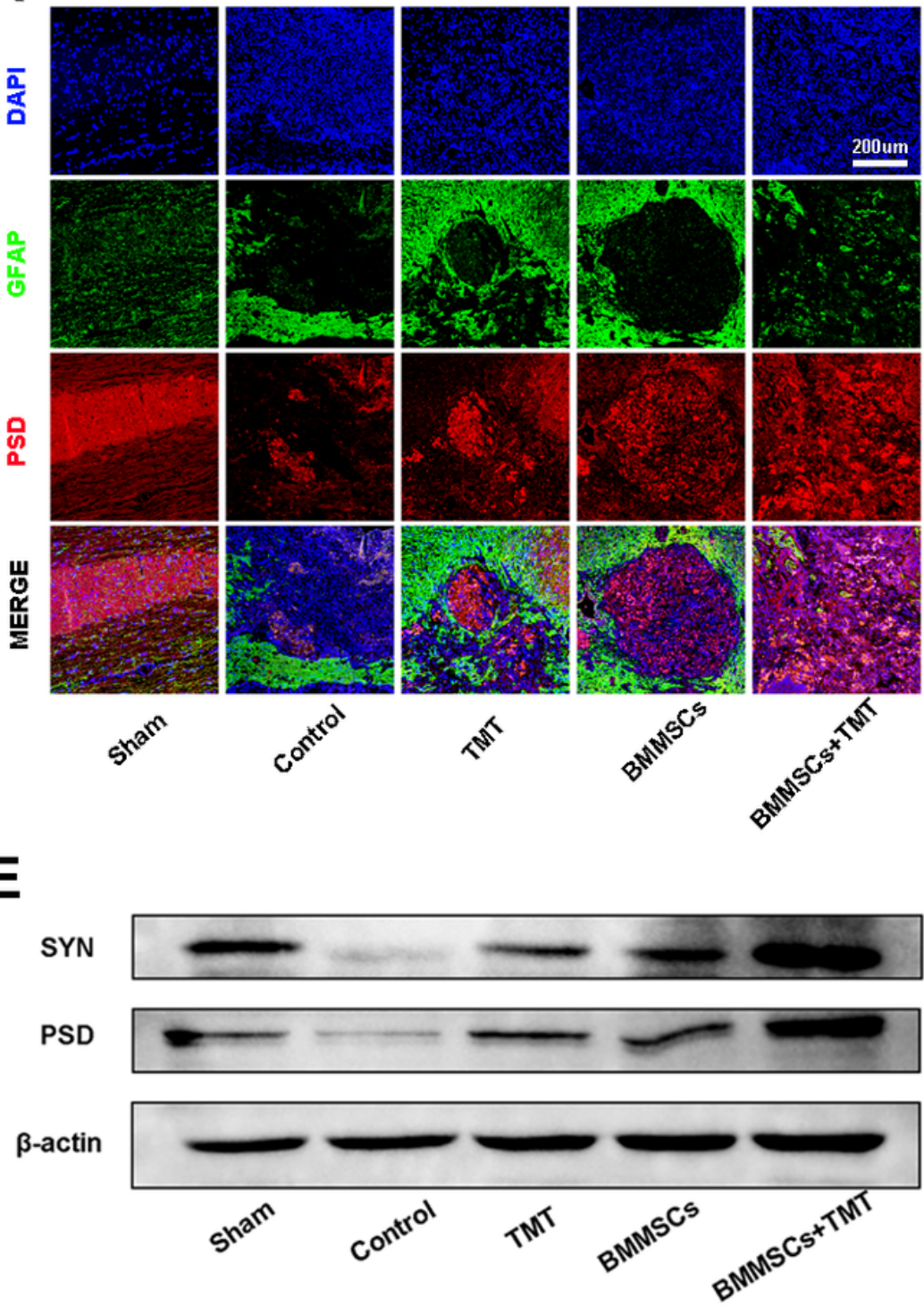

B
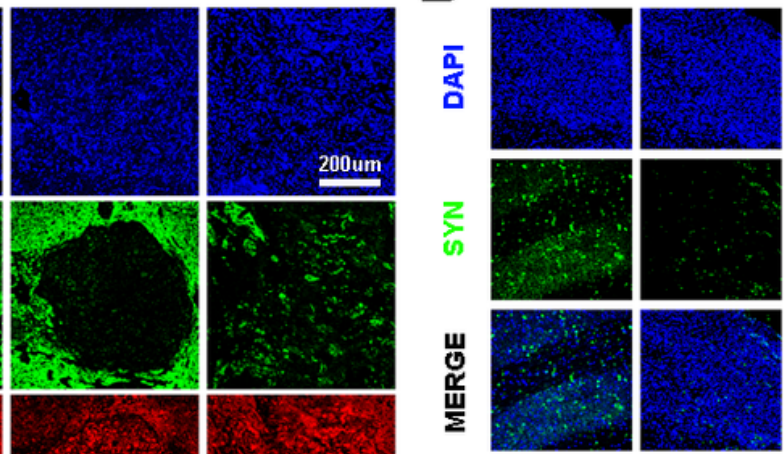

son

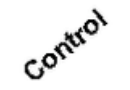

C

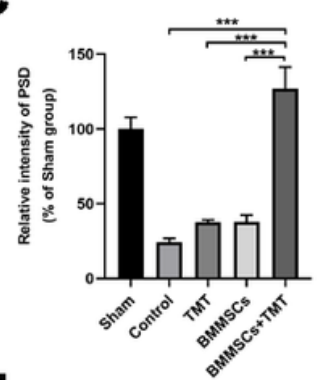

$\mathbf{F}$

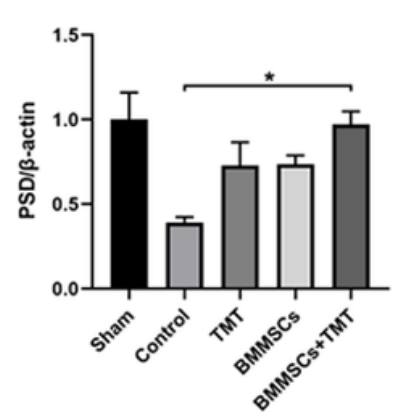

G
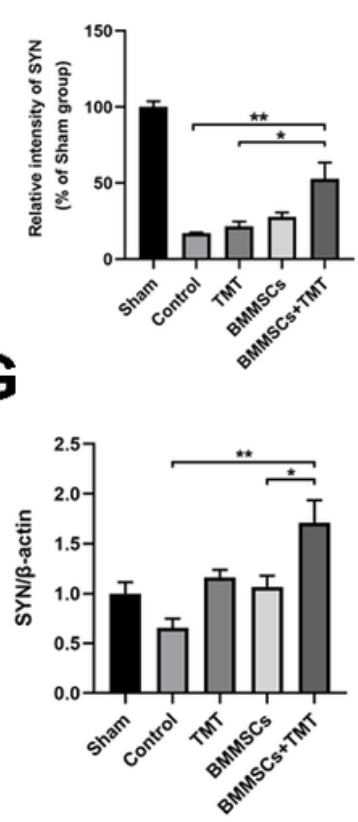

Figure5

\section{Figure 5}

Combining BMMSCs and TMT increased synaptic plasticity after SCI. A-B. Representative Images of immunofluorescence staining of PSD and SYN in the lesion center 8 weeks after SCl. GFAP_(green), PSD_(red), SYN_(red). C-D. Quantification of immunofluorescence staining of PSD and SYN. E-G. Representative western blot images and quantification of PSD and SYN. ${ }^{*} p<0.05,{ }^{*} p<0.01,{ }^{* \star *} p<0.001$. $\mathrm{N}=3$ per group. 
A

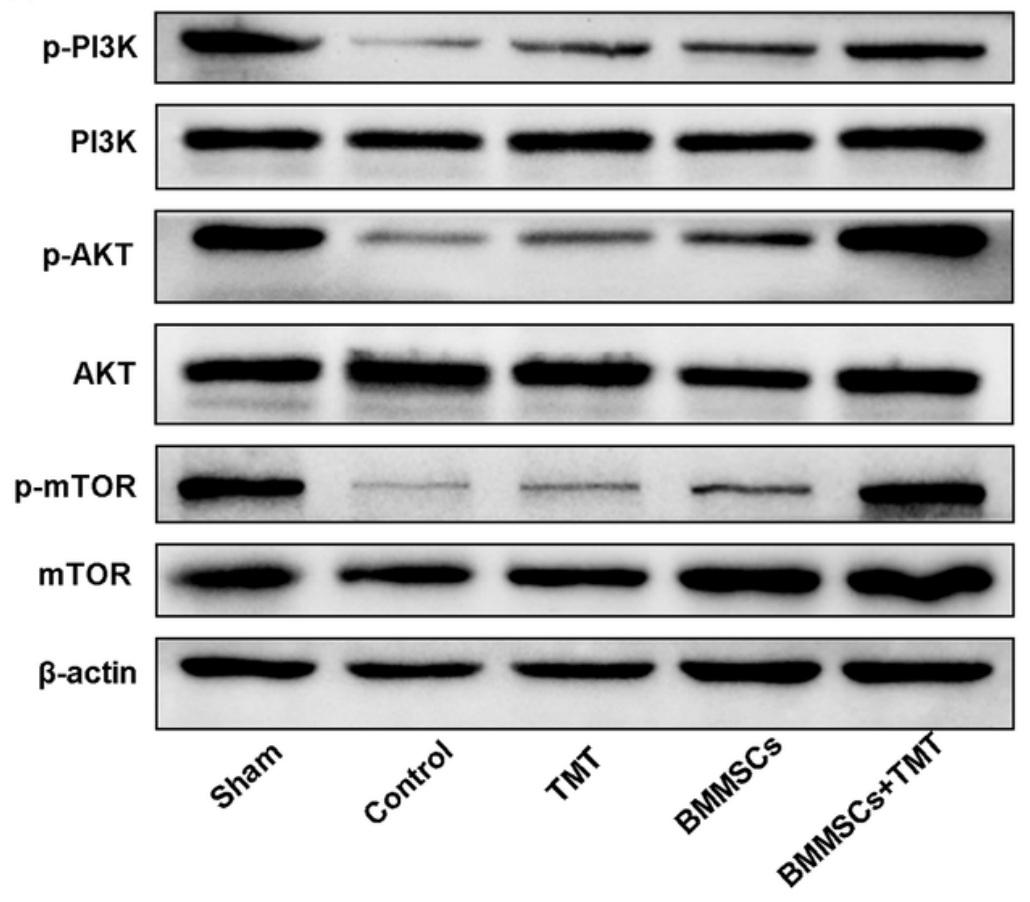

B

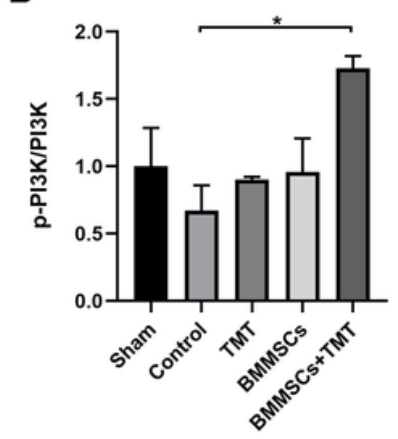

C

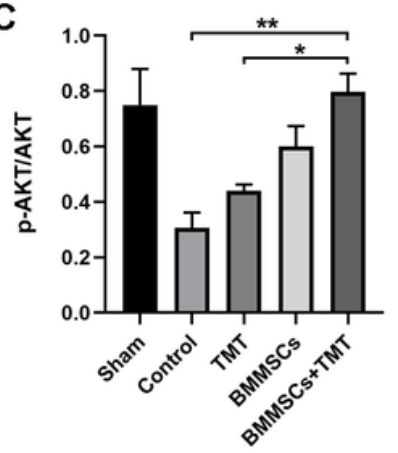

D

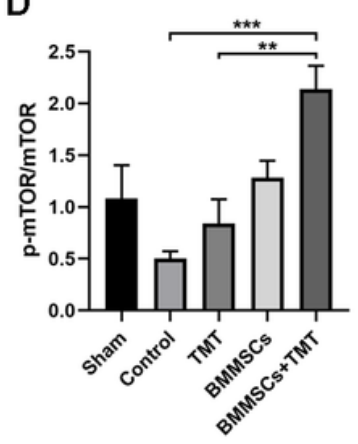

Figure6

Figure 6

Combining BMMSCs and TMT upregulated PI3K/Akt/mTOR signaling pathway. A. Representative western blot images of p-PI3K and PI3K, p-AKT and total AKT, p-mTOR and total m-TOR. B-D. The relative intensity of $p-P I 3 K / P I 3 K, p-A K T / A K T$ and $p-m T O R / m-T O R$ by western blot analysis. ${ }^{*} p<0.05,{ }^{* *} p<0.01$, $\star * \star p<0.001 . N=3$ per group.

\section{Supplementary Files}

This is a list of supplementary files associated with this preprint. Click to download.

- Additionalfile1.tif

- Additionalfile2.tif 